\section{Facharztprüfung zur Erlangung des Facharzttitels FMH für Pneumologie}

Aufgrund des Weiterbildungsprogrammes, welches am 1. Januar 2001 in Kraft gesetzt wurde, ist die Teilnahme an der Facharztprüfung für diejenigen Kandidatinnen und Kandidaten Voraussetzung für die Erlangung des Facharzttitels FMH für Pneumologie, welche ihre Weiterbildung in Pneumologie am 30. Juni 1997 nicht abgeschlossen hatten. Es empfiehlt sich, die Facharztprüfung frühestens im letzten Jahr der reglementarischen Weiterbildung abzulegen (Art. 22 WBO).
Datum: Donnerstag, 21. Juni 2001

Ort: Lungenliga Schweiz, Südbahnhofstrasse 14c, 3000 Bern 17

Prüfungsgebühr: Die SGP erhebt eine Prüfungsgebühr von Fr. 650.-.

Anmeldung: unter Beilage des Curriculums an folgende Adresse: Prof. Dr. med. Matthias Gugger, Pneumologische Abteilung, Inselspital, 3010 Bern.

Anmeldeschluss: 15. März 2001

Der Stoffkatalog kann bezogen werden beim Sekretariat der Schweizerischen Gesellschaft für Pneumologie (SGP), Südbahnhofstrasse 14c, Postfach 49, 3000 Bern 17, Tel. 03137820 30, Fax 03137820 31, E-mail: k.lehmann@lung.ch

\section{Facharztprüfung zur Erlangung des Schwerpunktes Pädiatrische Pneumologie zum Facharzttitel FMH für Kinder- und Jugend- medizin}

Aufgrund des Weiterbildungsprogrammes, welches am 1. Juli 1996 in Kraft gesetzt wurde, ist die Teilnahme an der Facharztprüfung für diejenigen Kandidatinnen und Kandidaten Voraussetzung für die Erlangung des Schwerpunktes Pädiatrische Pneumologie zum Facharzttitel FMH für Kinder- und Jugendmedizin, welche ihre Weiterbildung in Pädiatrischer Pneumologie bis am 30. Juni 1997 nicht abgeschlossen hatten. Es empfiehlt sich, die Facharztprüfung frühestens im letzten Jahr der reglementarischen Weiterbildung abzulegen (Art. 22 WBO).
Datum: Donnerstag, 21. Juni 2001

Ort: Lungenliga Schweiz, Südbahnhofstrasse 14c, 3000 Bern 17

Prüfungsgebühr: Die SGP erhebt eine Prüfungsgebühr von Fr. 650.-.

Anmeldung: unter Beilage des Curriculums an folgende Adresse: Prof. Dr. med. Markus Rutishauser, Blauenweg 1, 4102 Binningen

Anmeldeschluss: 15. März 2001

Der Stoffkatalog kann bezogen werden beim Sekretariat der Schweizerische Gesellschaft für Pneumologie (SGP), Südbahnhofstrasse 14c, Postfach 49, 3000 Bern 17, Tel. 03137820 30, Fax 03137820 31, E-mail: k.lehmann@lung.ch. 